\title{
Case report and review of the literature: Rectal linitis plastica secondary to the lipoid cell variant of transitional cell carcinoma of the urinary bladder
}

\author{
Victor A. McPherson, MD; ${ }^{*}$ Michael Ott, MD, FRCSC; ${ }^{\dagger}$ Edward J. Tweedie, MD, FRCPC, Jonathan I. Izawa, \\ $M D, F R C S C^{*}$
}

*Departments of Surgery \& Oncology, Divisions of Urology \& Surgical Oncology, The Schulich School of Medicine \& Dentistry, Western University, London, ON; †Department of Surgery, Division of General Surgery \& Surgical Oncology, The Schulich School of Medicine \& Dentistry, Western University, London, ON; §Department of Pathology, The Schulich School of Medicine \& Dentistry, Western University, London, ON

Cite as: Can Urol Assoc J 2012;6(6):431-4. htrp://dx.doi.org/10.5489/cuai.11239

\section{Abstract}

The overall 5-year survival of patients with urothelial carcinoma of the bladder (UC) is about $78 \%$; however, there are some rare subtypes. One of these is the lipoid cell subtype, which bears a very poor prognosis. Another rare disease entity with a poor prognosis is metastasis to the lower gastrointestinal tract in the form of secondary linitis plastica of the rectum. We describe an extremely rare and unique case of rectal linitis plastica secondary to the rare lipoid cell variant of UC.

\section{Introduction}

There are a variety of urothelial carcinoma of the bladder (UC) subtypes and many bear poor prognosis compared to conventional UC. ${ }^{1}$ One such rare variant is the lipoid cell subtype described in only 33 patients. ${ }^{1-3}$ The lipoid cell population typically composes $10 \%$ to $50 \%$ of the tumour, with the remainder being conventional $U \mathrm{C}$ or another rare subtype, including plasmacytoid ${ }^{3}$ and micropapillary. ${ }^{3,4}$ Lipoid cell $\cup C$ has a very poor prognosis, with disease-related death or patients living with disease in 31/33 cases, $^{1-3}$ compared with an overall 5 -year survival of about $78 \%$ seen in UC. ${ }^{5}$

Metastasis to the lower gastrointestinal tract $(\mathrm{LGI})$ tract is rare. A study reported an incidence of only 9/16 838 colonoscopies. ${ }^{6}$ Another study showed a prevalence of $6 / 598$ patients having rectal wall metastasis in those undergoing LGI-endoscopic ultrasound (EUS) to evaluate assumed primary rectal cancer. ${ }^{7} \mathrm{~A}$ rare $\mathrm{Gl}$ metastatic subtype is linitis plastica, characterized by circumferential intramural tumour infiltration of a hollow viscus, generating a desmoplastic reaction which results in a thickened, inelastic wall. ${ }^{8}$ This process can be either primary or secondary to metastasis, and involves the submucosa and muscularis propria, while sparing the mucosa. ${ }^{8}$ Diagnosis is often delayed, as the dis- ease shares imaging characteristics with other disease processes, and endoscopic biopsies are often negative due to mucosal sparing. ${ }^{8}$ Rectal linitis plastica (RLP) secondary to bladder cancer is extremely rare, ${ }^{6-9}$ and only one case report focuses on this disease. ${ }^{8}$

Both secondary RLP ${ }^{6,8}$ and the lipoid cell $U C^{1-3}$ have a poor prognosis; we have limited information on treatment response. We present a unique case of RLP secondary to lipoid variant UC.

\section{Case report}

A 67-year-old man presented with a 3-week history of diarrhea, fecal incontinence, constitutional symptoms and minor dysuria. On exam, there was a palpable fixed, circumferential, rectal mass with anal involvement into the scrotum. The patient had a history of hypertension, degenerative disk disease, and a 50-pack-year history of smoking and was not on medications.

Initial blood work showed a normal complete blood count, serum creatinine of $924 \mu \mathrm{mol} / \mathrm{L}$ and potassium of $7.9 \mathrm{mmol} / \mathrm{L}$. Carcinoembryonic antigen was $1.2 \mu \mathrm{g} / \mathrm{L}$ (reference range: 0-3.0) and prostate-specific antigen was $2.91 \mu \mathrm{g} / \mathrm{L}$. Urinalysis, and urine and stool cultures were negative. Renal ultrasound showed bilateral hydroureteronephrosis without urinary retention in the bladder. Bilateral nephrostomy tubes were inserted.

Once the creatinine normalized, contrast enhanced computed tomography (CT) of the abdomen and pelvis was performed, which showed asymmetric thickening of the left lateral and posterior bladder walls and diffuse $1.5-\mathrm{cm}$ thickening of the rectum and sigmoid colon from the anorectal junction to the mid-sigmoid (Fig. 1). Other findings included anasarca, perirectal and retroperitoneal edema and trace pelvic free fluid.

A magnetic resonance imaging (MRI) with gadolinium showed additional findings of an infiltrated colonic submucosa, markedly widened with minimal perirectal fat plane 

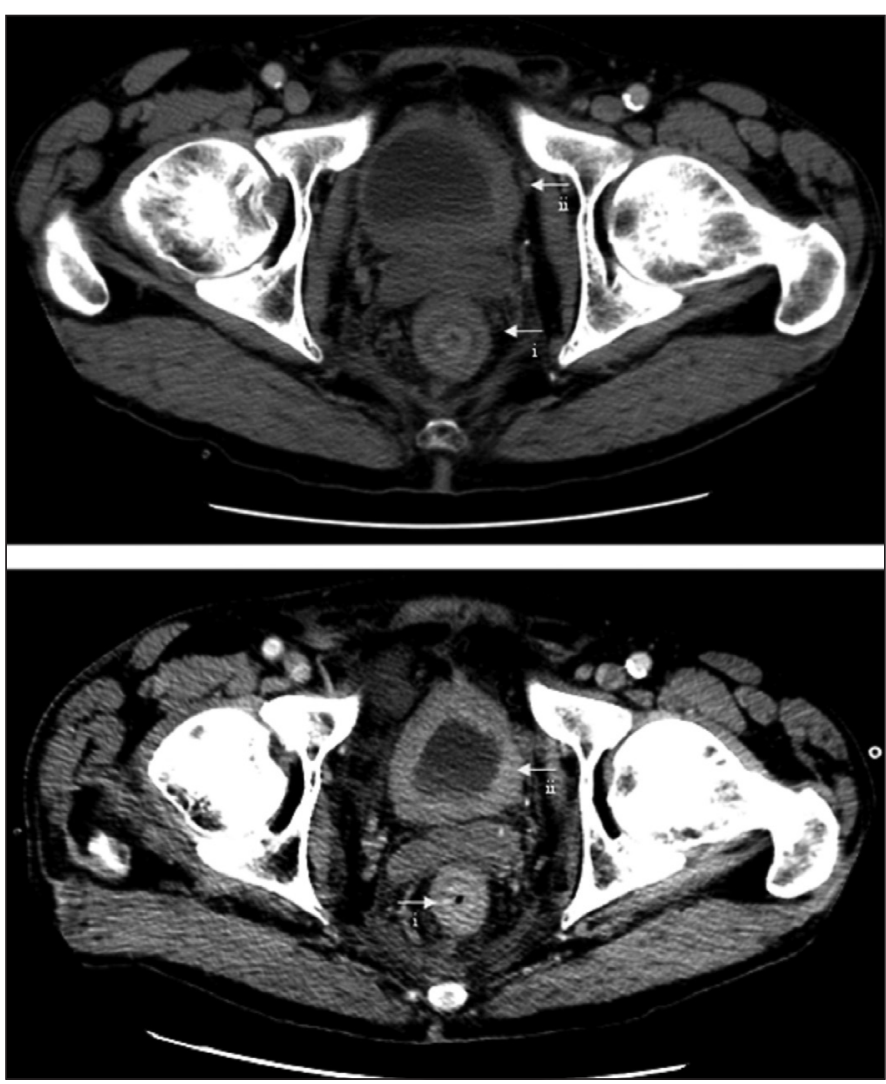

Fig. 1. Computed tomography (CT) imaging showing a primary bladder lesion and secondary rectal linitis plastica (RLP). A. CT image from Day 7 after presentation to the emergency room. Arrows denote: i) Concentric ring pattern of intramural thickening, wall $1.5 \mathrm{~cm}$ thick, characteristic of RLP. The lesion was noted to extend from the anorectal junction to the mid-sigmoid colon; ii) Posterior and left sided bladder wall thickening indicating a large mass in the bladder wall. B. CT image from day 53. Arrows denote: i) Concentric ring pattern of rectal wall thickening indicative of RLP; ii) Concentric thickening of the bladder wall indicating tumor progression compared to the previous CT.

infiltration (Fig. 2), consistent with rectal linitis plastica. The left seminal vesicle showed an infiltration of low signal, which was noted as likely secondary to peritumoral fibrosis.

A colonoscopy was performed using an ultra-thin colonoscope due to rectal obstruction. The mass showed no ulceration, erythema or polyps, but looked malignant. Eight core biopsies were taken and only one sample core contained atypical cells, which were suspicious for malignancy.

Urine cytologies were performed with two of three samples showing atypical cells, and cystoscopy identified a large, sessile bladder tumour on the left lateral wall and transurethral resection of bladder tumour was performed. The pathological examination showed an undifferentiated high-grade UC with muscularis propria invasion and showing sarcomatoid differentiation.

The patient had increasing, severe abdominal and rectal pain and failure to thrive. Plans were made to proceed with pelvic exenteration to at least palliate the patient's symptoms. The patient was admitted to hospital for a large bowel

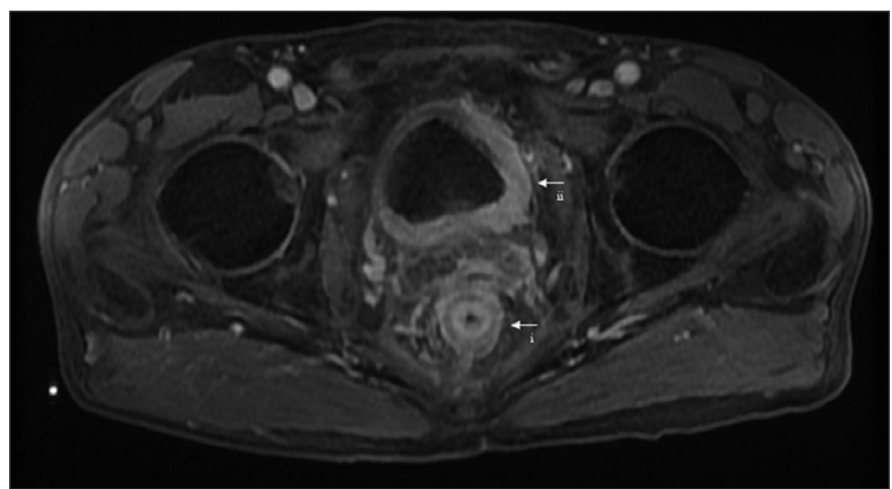

Fig. 2. Liver acquisition with velocity acceleration (LAVA) sequence magnetic resonance imaging (MRI) with gadolinium of the primary bladder lesion and secondary rectal linitis plastica (RLP). Arrows indicate: i) Concentric ring pattern of intramural thickening consistent with RLP, involving the colonic submucosa ii) Posterior and left lateral sided bladder wall thickening indicating a large mass within the bladder wall.

obstruction and had total parenteral nutrition due to his malnourished state. He underwent an abdominal perineal resection, resection of base of scrotum, total mesorectal excision, resection of small bowel, pelvic lymphadenectomy and ileal conduit diversion. Ascites and peritoneal carcinamatosis on the small bowel were found intraoperatively.

Gross pathological analysis identified a firm circumferential tan-white tumour measuring $9.8 \times 4.5 \times 1.1 \mathrm{~cm}$ occupying the entire urinary bladder. The tumour invaded the perivesicular fat and $15.5 \mathrm{~cm}$ of the bowel wall. The involved section of the bowel was stenosed, but contained grossly normal mucosa.

Microscopic analysis showed diffuse high-grade, poorly differentiated carcinoma, while a minority of cells contained large clear vacuoles, reflective of lipoid variant UC (Fig. 3). The tumour was found to be CK AE1/AE3 ${ }^{+v e}$, CK 34BE12 ${ }^{\text {+ve }}$, $\mathrm{CK} 7^{\text {+ve }}$, and $\mathrm{CK} 20^{\text {+ve }}$, while it was P63-ve ${ }^{\text {-ve }}$ CDX2-ve and vimentin $^{-v e}$. Additionally, there was an incidental focus of Gleason 6 prostate cancer incorporating $2 \%$ of the prostate.

In the bladder, the tumour grew in sheets, cords or as single cells and invaded the lymphatic space. The tumour invaded through the bladder wall and into the perivesicular and peri-prostatic fat. The tumour also showed diffuse infiltration into the wall of the adherent segment of rectum, while the mucosa was normal. The tumour also involved the perianal area, scrotal base and periurethral tissue. Multiple surgical margins and 3 lymph nodes were positive for malignancy. Additionally, a section of the small bowel was positive for malignancy, confirming carcinomatosis.

Initially the patient's wounds healed well and his pain was palliated, but the postoperative course was complicated by a large pelvic abscess that eroded into the ileal conduit and biliary obstruction caused by a metastatic lymph node. Additionally, a bone scan revealed a metastatic focus within the vertebral body of T6. The patient died 4 months after his initial presentation. 


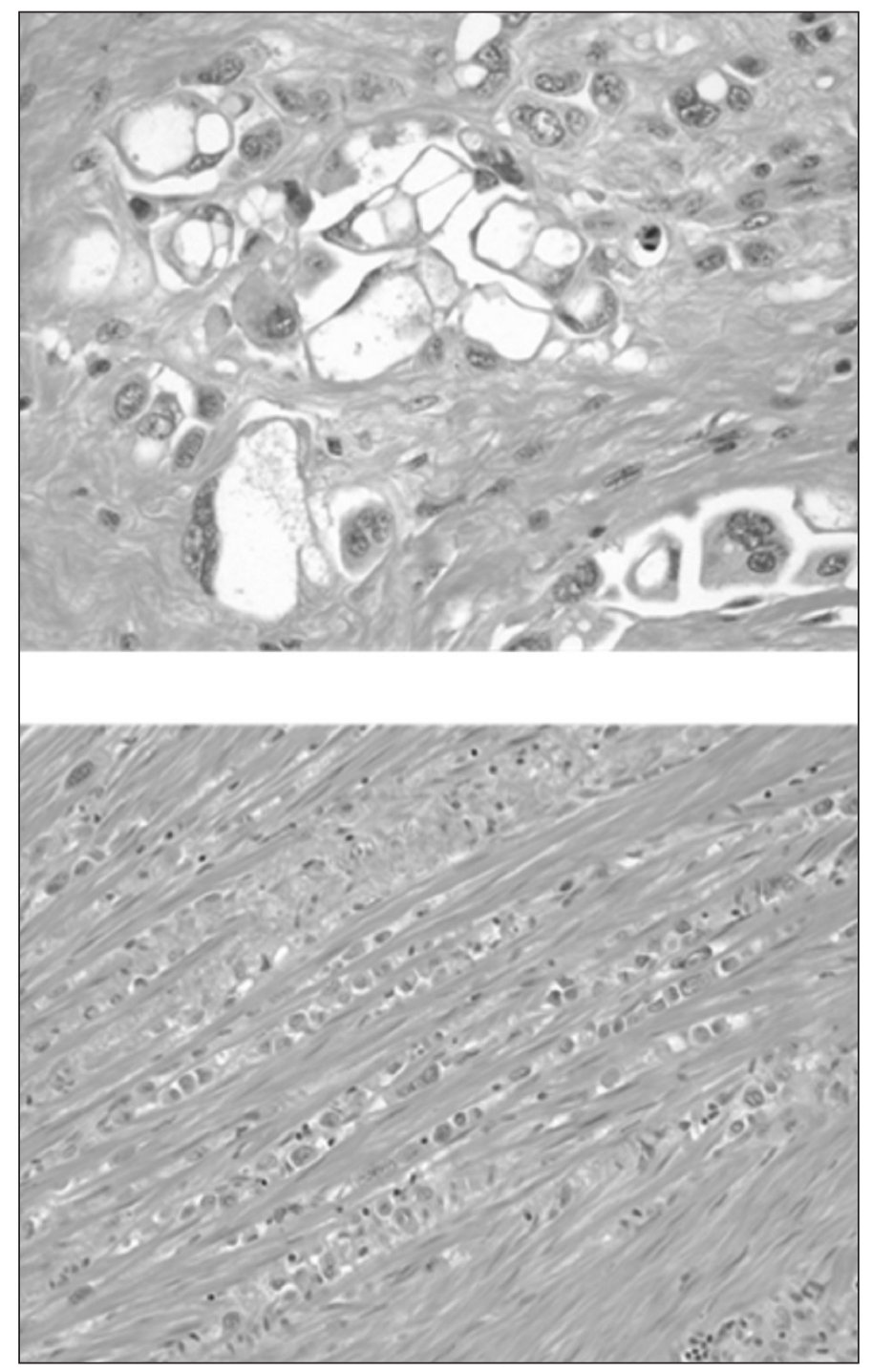

Fig. 3. Histological images of the lipoid cell variant of transitional cell carcinoma. A. Tumour cells infiltrating the bladder wall. Many cells have a lipoblast-like appearance with intracytoplasmic vacuoles which are mucin negative (40x magnification, hematoxylin and eosin). B. Tumour cells diffusely infiltrating the rectal wall (20x magnification, hematoxylin and eosin).

\section{Discussion}

Independently, the lipoid cell variant of UC and secondary RLP is rare and has a poor prognoses. Our patient's course reflected the expected aggressiveness of his combined disease.

No previous reports on the lipoid cell variant of UC have reported a linitis plastica tumour morphology..$^{2-4}$ The two previous cases of RLP secondary to UC with reported pathology showed conventional UC in 1 patient and papillary variant UC containing a component of adenocarcinoma in the other. ${ }^{8}$ Thus, there does not appear to be a specific UC subtype that is predisposed to presenting as linitis plastica.

Our case of secondary RLP was consistent with previous reports in that significant circumferential rectal stenosis was encountered with LGI endoscopy. ${ }^{7,8}$ In addition, our endoscopic biopsies were inconclusive for malignancy, which is a common finding in $\mathrm{RLP},{ }^{7,8}$ as the disease primarily involves the submucosa and muscularis propria while sparing the mucosa.

Evidence suggests that the imaging modality of choice for diagnosing secondary RLP is endoscopic ultrasound (EUS). Studies have shown that EUS often shows local-regional tumour involvement that is not found by $\mathrm{CT},{ }^{10}$ and can detect RLP missed by CT or MRI. ${ }^{7}$ In our case, EUS imaging was not done. Locoregional involvement shown by CT in our case included mild anasarca with free fluid in the pelvis and edema through the retroperitoneal fat plane. MRI noted minimal infiltration in the perirectal fat plane and lymphadenopathy within the mesorectum, with the largest lymph node having a short axis measuring $5 \mathrm{~mm}$. While it is unknown if these findings would have been earlier identified using EUS, it is unlikely to have changed the palliative management.

A key delineation in our case involved the differentiation between lipoid cell UC and signet ring cell carcinoma (SRC). SRCs in the Gl tract most often produce a primary linitis plastica tumour morphology and are frequently documented in primary $R L P, 11$ and infrequently in primary linitis plastica of the bladder. ${ }^{12}$ SRCs are composed of signet ring cells, displaying large mucin containing intracellular vacuoles; signet ring cells are morphologically similar to UC lipoid cells. ${ }^{2-4}$ However, SRCs are composed entirely of signet ring cells, while lipoid cell $U C$ is always associated with another UC variant. ${ }^{2-4}$ Most commonly, lipoid cell $U C$ is associated with conventional UC, with lipoid cells composing $10 \%$ to $50 \%$ of the tumour cell population. ${ }^{3}$ These lipoid cells resemble lipoblasts, as they have several large intracellular vacuoles (Fig. 3). There are also several key differences in immunohistochemical staining patterns of lipoid cells and signet ring cells. Lipoid cells often stain $\mathrm{CK} 7^{\text {+ve }} / \mathrm{CK} 2 \mathrm{O}^{+\mathrm{ve}}{ }^{2-4}$ while a study found that only 10/60 SRCs of colonic, gastric and breast origin stained $\mathrm{CK}^{+\mathrm{ve}} / \mathrm{CK} 2 \mathrm{O}^{+\mathrm{ve}} \cdot{ }^{13}$ Additionally, this study found that $4 / 5$ SRCs of colorectal origin stained CDX2 ${ }^{+v e} \cdot{ }^{13}$ Finally, mucin production is characteristic for SRCs, ${ }^{13}$ while none of the documented lipoid cell UC cases tested have stained positively for mucin, ${ }^{3}$ including our own. Thus, our patient's tumour was consistent with lipoid cell UC.

Lipoid variant UC has an extremely poor prognosis, ${ }^{2-4}$ even in the absence of RLP. The maximum documented survival has been 76 months, ${ }^{2}$ with one study reporting that 16/27 patients died of their disease (DOD), while 10/27 were alive but with disease (AWD) recurrence at time of publication. ${ }^{3}$ Of the remaining documented cases, 5/6 resulted in patient mortality. ${ }^{2,4}$

Lopez-Beltran and colleagues reported the mean time to death for patients was 33 months, and mean time to recurrence was 22 months. ${ }^{3}$ Of these patients, 17 underwent 
cystoprostatectomy \pm radiation or chemotherapy, while the remaining 10 patients underwent transurethral resection of bladder tumour (TURBT) \pm bacillus Calmette-Guerin (BCG). ${ }^{3}$ The remaining 5 reported patients who died had a mean survival of 25 months. ${ }^{2,4}$

In a case report by Soylu and colleagues, the patient had clinically defined $\mathrm{T} 2 \mathrm{bNxM0}$ at presentation, received non-standard 5-fluorouracil/leukovorin chemotherapy and survived for 18 months; ${ }^{4}$ Leroy and colleagues reported an average survival of about 25 months among their patients. ${ }^{2}$ The lone surviving patient reported by the authors received radical cystectomy without prior TURBT for pT2G3 disease, however, this patient only had 12 months of follow-up. ${ }^{2}$ The 4 remaining patients in this study received: (1) TURBT with BCG for pT1 G3 disease, DOD at 13 months; (2) TURBT with BCG and subsequent cystectomy for pT1 G3 disease, DOD at 76 months; (3) cystectomy for pT2 G3 disease, DOD at 24 months; and (4) nephrectomy with chemotherapy for pT3 G3 disease of the renal pelvis, DOD at 8 months.

In the study by Lopez-Beltran and colleagues, 3/27 patients had T1 or Ta disease and yet 26/27 cases suffered disease recurrence or death indicating that the T1 lipoid cell variant of UC tumours have a poor prognosis and should not be managed conservatively. ${ }^{3}$ Patients with early stage disease may benefit from early radical cystectomy rather than with TURBT and BCG. In contrast, patients with more advanced disease do not have an increased rate of cure with aggressive surgical management, but may have an increased length of survival and palliation.

RLP secondary to UC is also a rare disease entity, with a lone 2008 case report documenting the MRI findings in RLP secondary to UC. ${ }^{8}$ Two studies have reported cases while investigating the features of RLP secondary to generalized metastatic disease. ${ }^{7,9}$ One study reported metastatic lesions to the rectum from bladder cancer, but did not specify tumour morphology. ${ }^{6}$ None of the 43 patients (34 UGI, 9 LGI) with metastasis to the Gl tract reported on by Wei and colleagues survived beyond about 3.5 years. ${ }^{6}$ However, the group of patients that received surgical intervention, endoscopic hemostasis, interventional angiography or chemotherapy had a significantly longer survival than patients receiving conservative management. ${ }^{6}$ In another study, one of two patients with RLP secondary to bladder cancer was cured of their disease through the use of a combination of pelvic exenteration and neoadjuvant radiotherapy. ${ }^{\circ}$

\section{Conclusion}

These results support the use of aggressive surgical management to improve both the length and rate of survival in RLP secondary to bladder UC, while suggesting a role for neoadjuvant radiotherapy.

Competing interests: None declared.

This paper has been peer-reviewed.

\section{References}

1. Nigwekar P, Amin MB. The many faces of urothelial carcinoma: an update with an emphasis on recently described variants. Adv Anat Pathol 2008;15:218-33. http://dx.doi.org/10.1097/ PAP.0b013e31817d79b9

2. Leroy $X$, Gonzalez S, Zini L, et al. Lipoid-cell variant of urothelial carcinoma: a clinicopathologic and immunohistochemical study of five cases. Am J Surg Pathol 2007;31:770-3. http://dx.doi.org/10.1097/01. pas. 0000213410.48805 .16

3. Lopez-Beltran A, Amin MB, Oliveira PS, et al. Urothelial carcinoma of the bladder, lipid cell variant: clinicopathologic findings and LOH analysis. Am J Surg Pathol 2010;34:371-6. http://dx.doi.org/10.1097/ PAS.0b013e3181cd385b

4. Soylu A, Aydin NE, Y.lmaz U, et al. Urothelial carcinoma featuring lipid cell and plasmacytoid morphology with poor prognostic outcome. Urology 2005;65:797. http://dx.doi.org/10.1016/i.urology.2004.10.005

5. Mungan NA, Aben KK, Schoenberg MP, et al. Gender differences in stage-adjusted bladder cancer survival. Urology 2000;55:876-80. http://dx.doi.org/10.1016/S0090-4295(00)00523-9

6. Wei SC, Su WC, Chang MC, et al. Incidence, endoscopic morphology and distribution of metastatic lesions in the gastrointestinal tract. J Gastroenterol Hepatol 2007;22:827-31. http://dx.doi.org/10.1111/i.1440$1746.2006 .04532 x$

7. Gleeson FC, Clain JE, Rajan E, et al. Secondary linitis plastica of the rectum: EUS features and tissue diagnosis (with video). Gastrointest Endosc 2008;68:591-6. http://dx.doi.org/10.1016/i.gie.2008.04.027

8. Dresen RC, Beets GH, Vliegen RF, et al. Linitis plastica of the rectum secondary to bladder carcinoma: a report of two cases and its MR features. Br J Radiol 2008;81:e249-51. http://dx.doi.org/10.1259/ $\mathrm{bir} / 59924178$

9. Ha HK, Jee KR, Yu E, et al. CT features of metastatic linitis plastica to the rectum in patients with peritoneal carcinomatosis. AJR Am J Roentgenol 2000;174:463-6.

10. Dumontier I, Roseau G, Palazzo L, et al. Endoscopic ultrasonography in rectal linitis plastica. Gastrointest Endosc 1997;46:532-6. http://dx.doi.org/10.1016/50016-5107(97)70009-9

11. Kim HJ, Ha HK, Cho KS, et al. CT features of primary colorectal signet-ring cell carcinoma. J Comput Assist Tomogr 2001;25:225-30. http://dx.doi.org/10.1097/00004728-200103000-00011

12. Grignon DJ, Ro JY, Ayala AG, et al. Primary signet-ring cell carcinoma of the urinary bladder. Am J Clin Pathol 1991;95:13-20.

13. Chu PG, Weiss $L M$. Immunohistochemical characterization of signet-ring cell carcinomas of the stomach, breast, and colon. Am J Clin Pathol 2004;121:884-92. http://dx.doi.org/10.1309/ A09ERYMFR64NERDW

Correspondence: Dr. Jonathan I. Izawa, Departments of Surgery \& Oncology, Divisions of Urology \& Surgical Oncology, The London Health Sciences Centre-Victoria Hospital, 800 Commissioners Rd East, Room E2-649, London, 0N N6A 5Cl; jonathan.izawa@/hsc.on.ca 